\title{
Fiber Fly Generation of Soybean Yarns during Weaving
}

\section{Mehmet E Yuksekkaya*}

Department of Textile Engineering, Usak University, Usak 64200, Turkey

\begin{abstract}
At textile factories, fiber fly generation is still a major problem almost at every stage of productions. Therefore, it is a crucial factor to perform more studies of the fiber fly generation in respects to fiber types and production methods. Current study examines the fiber fly generation of soybean yarns during the weaving operation. Among all of fiber properties, the fiber mean length and fiber fineness have the greatest effects on the amount of the fiber fly generated during the processes. Yarn type and yarn twist level have also a significant effect on the fiber fly generations during the weaving.
\end{abstract}

Keywords: Soybean yarns; Fiber fly; Fly generation; Amount of fly; Yarn hairiness

\section{Introduction}

Despite the advances made in the field of textile machinery, fiber fly generation is still a major problem for textile industry not only for worker's health but also for quality problems of the products. The study of the fiber fly generations during manufacturing processes was studied by many researchers, especially after the intensive works have been performed on the physical properties and the hairiness of the yarns. The amount of the fiber fly generation is affected by many parameters, including yarn speed and fiber tenacity.

The soybean protein fiber is spun by using bio-engineering technology with natural soybean as a major raw material. In the process, globin is extracted and purified from the soybean meal, the spatial structure of which is modified to prepare protein spinning solutions. After curing, the wet spinning technique is employed to spin fibers with the linear density of between 1.27-3.3 dtex, and by the performance of stable fiber of chemical cross-linking and through curving, heat setting and cutting, staple textile fibers are produced of various length specifications. Currently, it is one of the most comfortable raw materials of textile fibers, and also called artificial cashmere due to its extraordinary softness and comfort. Soybean protein fiber contains 18 amino acids necessary for the human body, is characterized by good affinity with skin, soft handle and superior penetrability, and especially demonstrates obvious advantages in knitting garments. With the functions and effects of bacterial inhibition, negative ions and resistance to ultraviolet, soybean fiber is an excellent material to fabricate top-grade knitting fabrics and household textile items.

The fiber fly generations are getting close attention as the production speeds of the machines are getting higher, which results more fiber fly generation at the working environment. It is being considered as a bigger problem than it used to be since it is becoming a mandatory regulation for textile mills to prepare a healthier and safer working environment for their employees [1-3]. Not only does the fiber fly generation cause an unhealthy working environment for people due to the intensity of the dust, but it also affects the quality of the products because of a possible contamination of the fiber fly to the products [1]. It may cause yarn breakages and mechanical defects in the machine during the weaving processes as well [4-7]. In order to prevent the effects of the fiber fly on the weaving mills, the whole production facility is usually equipped with air conditioning and filter systems. However, neither air conditioning nor filters are capable of reducing the total amount of the fiber fly generated during the manufacturing processes at the textile mills.
Studies showed that the fabrics produced from the hairy yarns would be capable of generating more fiber fly during the warp preparation and fabric dry finishing processes. Yuksekkaya [2] studied the fiber fly generation during the raising operation and reported that the amount of the fiber fly generated during rising related to the hairiness of the yarns used in the fabrics. Author also reported that during warp preparation, the vortex yarn was giving the least amount of the fiber fly [3]. Lawrence and Mohamed [5] studied the fiber fly distribution on the weft knitting machines. They reported the fiber fly distribution along the thread line according to the fiber length and percentage of fibers. In their studies, they also found that most of the short fibers were removed from the yarn at the first stage of the production.

There are some other parameters also affecting the amount of the fiber fly coming from the yarn during the spinning processes such as traveller weight, smoothness of the ring surface, and spindle speed for woolen yarns. Pillay [8] reported that high spindle speeds would give more hairiness for ring spun yarns, which cause more fiber fly generation at the further stages during the production. Furthermore, the amount of the draft ratios and number of drafting processes also influence the hairiness of the yarn in a way that high draft ratio increases the hairiness of the yarn on a single draw frame; however, as the number of drawing operations increases for the same amount of the draft ratio as in one passage draw frame, the hairiness of the yarn decreases. Since increasing the number of draw frames increases the attenuation of fibers in the yarn, the result will be fewer loose ends coming from the yarn surface which results in a reduction of the total mass of the fiber fly being produced during the weaving.

The fiber fly problem seems to be a difficult problem to be solved soon [9-12] but the textile machinery producers are trying to reduce the fiber fly generation as much as they can by adapting new technologies, which reduce the amount of stress and friction introduced into the yarns during the productions. This study is a continuation of previous studies examined the fiber fly generations at textile mills [13-16]. In this paper, particularly, the fiber fly generations during weaving were

*Corresponding author: Mehmet E Yuksekkaya, Department of Textile Engineering, Usak University, Usak, Turkey, E-mail: mehmetemin.yuksekkaya@usak.edu.tr

Received November 29, 2012; Accepted December 27, 2012; Published January 04, 2012

Citation: Yuksekkaya ME (2012) Fiber Fly Generation of Soybean Yarns during Weaving. J Textile Sci Eng 2:124. doi:10.4172/2165-8064.1000124

Copyright: (C) 2012 Yuksekkaya ME. This is an open-access article distributed under the terms of the Creative Commons Attribution License, which permits unrestricted use, distribution, and reproduction in any medium, provided the original author and source are credited. 
studied emphasizing the role of fiber properties, environmental factors, the influences of yarn spinning parameters for the yarns spun from soybean fibers, and the effects of the different parts on the weaving machines by using ring and open end yarns.

\section{Material and Methods}

In this study, weaving heavy fabrics were chosen to compile the fiber fly generation at real time by using yarns spun from the soybean fibers. The amount of the fiber fly generation for a single yarn end is very low and not an easy task to detect accurately most of the time. Therefore, it is necessary to collect intensive measurements in order to have an accurate and a valid test results. The test data were collected from a commercial weaving factory that is using ring and open end yarns in its regular production. One of the weaving machines, Smit GS900 2005 model, at the site was chosen and modified in order to collect the fiber fly easily for this research according to the previous studies $[2,3,12]$ as described below:

The modification on the weaving machine was only made to collect the fiber fly easily and accurately by using filters during the process. Specially designed vacuum orifices have been placed at the fiber fly collection points. The orifices, then, were connected to the suction device equipped with a filter and hose to collect the entire fiber fly generated during the weaving. The suction units separately installed to every data collection point and could work independently from the other suction units. The modification made to the loom was not affecting the working conditions of the machine.

The previous studies have shown that the yarn speed is one of the major factors affecting the fiber fly generation $[5,2,3,12]$. The selected loom was working at around 360 picks/min with 18 picks/ $\mathrm{cm}$. The sizing of warp yarns helps to reduce the amount of the fiber fly generation due to the friction between yarn and reed. Therefore, the data were only collected from the weft yarns in this study. The main characteristics of weft yarns used in the weaving operations are as follows: Three different yarns, namely 200, 250, and 300 tex open end and ring yarns have been chosen for the experimental purposes. There were four different coefficient of twist associated with the spun yarns, namely, $\alpha_{\text {tex }}=22.13(\alpha \mathrm{m}=70), \alpha_{\text {tex }}=25.30 \quad(\alpha \mathrm{m}=80), \alpha_{\text {tex }}=28.46$ $(\alpha \mathrm{m}=90)$, and $\alpha_{\text {tex }}=31.62(\alpha \mathrm{m}=100) \frac{\text { turns }}{\mathrm{cm}} \sqrt{t e x}$ twist multipliers were used in the experiment. The yarn counts, yarn types, and the twist levels were the standard parameters that the producer was using in their regular productions associated with the required properties of the end products.

Twelve measurements have been taken for each combination of the test with $12.5 \mathrm{~kg}$ of yarns. Totally, $150 \mathrm{kgs}$ of yarns have been used for each combination of the test in the experiment in order to gain accurate measurements. The collected fiber fly was weighted by using a laboratory type scale. The amount of the fiber fly was, then, normalized by dividing the amount of the fiber fly in grams to the weight of the yarn in kilograms in order to make the comparison more logical for each level of trials. Table 1 shows the statistics of the fiber fly generation test.

\section{Factors Influencing Fiber Fly Generation}

Most of the time, blanket fabrics are woven by using the jacquard or dobby looms. Depending on the applications, the warp yarns are in the order of 30 tex with $\alpha_{\text {tex }}=42.66\left(\alpha_{\mathrm{m}}=120\right) \frac{\text { turns }}{\mathrm{cm}} \sqrt{\text { tex }}$ twist multiplier and sized in order to minimize the warp breakage and increase the

\begin{tabular}{|c|c|c|c|c|c|c|c|c|c|}
\hline \multirow[t]{2}{*}{$\begin{array}{l}\text { Yarn } \\
\text { Type }\end{array}$} & \multirow{3}{*}{\begin{tabular}{|l|} 
Yarn \\
Count \\
(Tex)
\end{tabular}} & \multicolumn{8}{|c|}{ Twist Factor $\left(\frac{t}{c m} \sqrt{t e x}\right)$} \\
\hline & & \multicolumn{2}{|c|}{22.13} & \multicolumn{2}{|c|}{25.30} & \multicolumn{2}{|c|}{28.46} & \multicolumn{2}{|c|}{31.62} \\
\hline \multirow[t]{4}{*}{$\begin{array}{l}\text { Ring } \\
\text { Yarn }\end{array}$} & & $\begin{array}{c}\text { Average } \\
\text { (gr/kg) }\end{array}$ & CV \% & $\begin{array}{c}\text { Average } \\
\text { (gr/kg) }\end{array}$ & CV \% & $\begin{array}{c}\text { Average } \\
\text { (gr } / \mathrm{kg})\end{array}$ & CV \% & $\begin{array}{c}\text { Average } \\
\text { (gr/kg) }\end{array}$ & CV \% \\
\hline & 200 & 13.79 & 6.91 & 11.18 & 8.03 & 10.29 & 9.61 & 9.27 & 9.64 \\
\hline & 250 & 16.23 & 9.19 & 11.09 & 8.42 & 9.18 & 8.06 & 6.56 & 9.43 \\
\hline & 300 & 15.20 & 9.64 & 10.06 & 9.09 & 8.37 & 9.76 & 5.90 & 8.01 \\
\hline \multirow{3}{*}{$\begin{array}{l}\text { Open } \\
\text { End } \\
\text { Yarn }\end{array}$} & 200 & 9.13 & 9.79 & 6.78 & 8.74 & 5.84 & 7.49 & 3.82 & 9.79 \\
\hline & 250 & 10.06 & 9.17 & 7.56 & 9.72 & 6.26 & 9.80 & 4.48 & 9.41 \\
\hline & 300 & 10.67 & 9.34 & 7.87 & 8.69 & 6.51 & 9.59 & 4.96 & 9.51 \\
\hline
\end{tabular}

Table 1: Amount of fiber fly generation on measured samples.

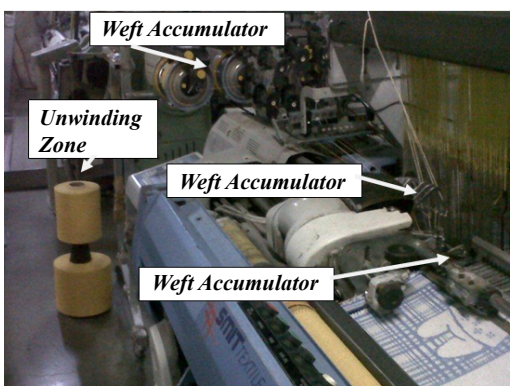

Figure 1: Effects of Moisture Contents on Fiber Fly Generations.

weaving efficiency. However, the weft yarns are coarser and have a lower twist factor compared to the warp yarns. Since the speed of the weft yarn is faster and the twist factor is lower, the majority part of the fiber fly generated during the weaving is coming from the weft yarns.

\section{Impacts of moisture content}

During the weft insertion, the weft yarns were subjected to some amount of stress that results into friction, which causes the fiber, fly generation. To overcome this continuous wear, it is necessary to supply sufficient amount of humidity to the weaving house. The moisture rate of the yarn is, therefore, another factor influences the fiber fly generation during the weaving. In the experiment, the yarns were kept at the conditioning chamber for at least 48 hours. As seen in figure 1 , as the moisture content of the yarn increases, the amount of the fiber fly decreases. Increasing the moisture content results in a swelling of the fibers and provides a higher amount of friction between the fibers. Therefore, it is an important factor to control the moisture contents of the yarn during the weaving process in order to have a less amounts of the fiber fly generation.

\section{Impacts of fiber properties}

It is well known that the fiber properties have significant effects on the fiber fly generations for staple yarns, mostly due to the variation in the physical properties of the yarns. In order to determine the effect of fiber fineness and fiber mean length, the yarn count and the twist level should be kept constant. It was reported that the fiber length had the greatest effect on the fiber fly generation during knitting and warp preparation than any other fiber properties [5,7,2]. For the sake of this experiment, different fiber cut length was prepared by using a tow cutting machine. The effect of fiber mean length on the mass of the fiber fly generated during weaving is given in figure 2. As the mean fiber length increases, the total amount of the fiber fly generated during weaving decreases as seen in the figure. The longer fiber means length distribution aids frictional forces and results in better cohesion among 


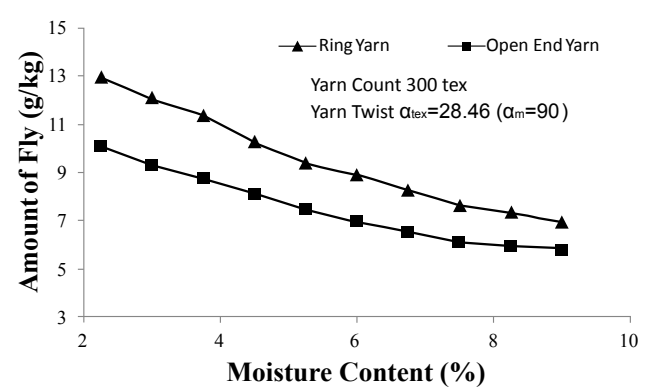

Figure 2: Effects of Fiber Mean Length on Fiber Fly Generations.

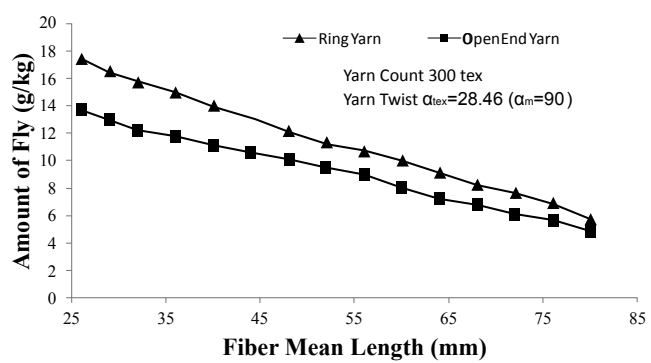

Figure 3: Effects of Fiber Linear Density on Fiber Fly Generations.

the fibers. Increasing the fiber mean length also reduces the number of loose ends that cause more fiber fly. The fiber fly generation during the weaving for open end yarns is getting lower as the mean length of fiber increases for a given yarn number. One reason for this reduction is the presence of the wrapper fibers in the yarn structure. As the fiber length increases, the number of wrapping coils also increases for a given yarn number. Thus, the fiber fly is getting lower significantly for open end yarns. Since the soybean fibers are artificially produced, it is possible to control the cutting length of the fibers during the fiber production in order to have a longer wrapper fiber in the yarn.

The linear density of fibers is another factor affecting the amount of the fiber fly generation during the weaving processes. For a given yarn count, the number of fibers in the cross-section of yarns depends on the fiber linear density. As the number of fibers increases in the yarn cross section, the hairiness of the yarn also increases [7,1,2,12]. These loose fiber ends generate fiber flies during the weaving process. Figure 3 shows the effects of fiber fineness on the fiber fly generation during the weaving operation. As seen in the figure, the amount of the fiber fly is higher for ring yarns than the rotor yarns. One reason for this phenomenon could be wrapper fibers. Since the wrapper fibers are protecting the core of open end yarns, the fiber fly generation is less than that of ring yarns regardless of the fiber linear density. It is also possible to see on the figure that as the fiber linear density increases, the amount of the fiber fly slightly increases due to the fact that the weight of the fiber per unit length gets higher for coarse fibers.

\section{Impacts of loom speed}

The pick insertion speed of the weaving machine has a great influence on the fiber fly generation during the weaving processes. As the loom speed increases, the amount of the fiber fly also increases. As seen in figure 4, up to 275 picks/min, the amount of the fiber fly increases slowly regardless of yarn types. Above $385 \mathrm{pick} / \mathrm{min}$, the amount of the fiber fly increases sharply especially for the ring yarns. One reason for this significant increase is that as the traveling speed of the yarn increases, the impact energy affecting to the fiber ends is getting severe. This increased energy cuts off the fibers protruding from the surface of the yarn and also wearing out the core fibers as explained in the previous studies $[3,12]$.

\section{Impacts of spinning types}

The past studies showed that there was a good correlation between the amount of the fiber fly generated during knitting [17]. For the current study, figure 5 shows the effect of spinning types on the fiber fly generated during the weaving. It could be seen from the figure that the ring yarns were giving the higher amount of the fiber fly than that of the open end yarns. The amount of the fiber fly is closely related to the structure of the yarns. It is widely known that open end yarns do not have a twist structure similar to that of ring yarns, yet, they have wrapper fibers on the surface which cover the core fibers. When this type of yarns comes in contact with machine parts, the wrapper fibers act as a shield to protect the core fibers. However, the wrapper fibers could be damaged at the initial contacts and produce the fiber fly. If the contact continues to wear the wrapper fibers, the core fibers are also damaged and began to generate the fiber fly as well. Table 2 shows the analysis of variances [18] data compiled by using SPSS program. As seen on the table, the ANOVA tests clearly indicate that the yarn type and the twist factor have a strong effect on the fiber fly generation while the yarn counts do not have a strong effect. Since yarn type and twist factor have significant effects, all the interactions between the factors are also significant for the fly generation.

The effect of twist level can be seen in figure 6 on the fiber fly generated during the weaving process. As the twist level increases, the amount of total fiber fly generated during the process decreases. The reason for this reduction is attributed to the increase of the frictional force among the fibers. Therefore, it is expected to have fewer amounts of the fiber fly when the twist level is higher as seen on the figure. Anova test is also indicated that the twist factor has significant effects on the amount of fiber fly generations.

Yarn linear density is another factor in the fiber fly generation. The

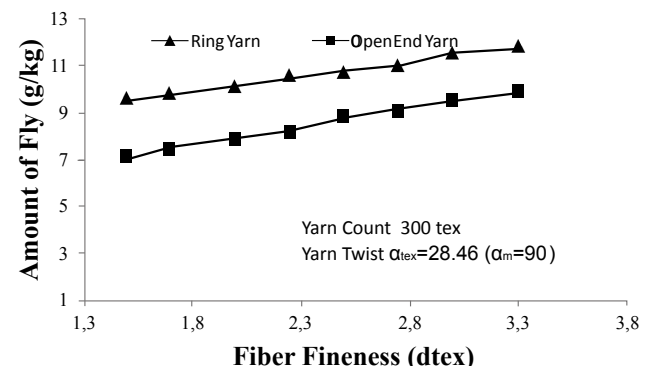

Figure 4: Effects of Loom Speed on Fiber Fly Generations.

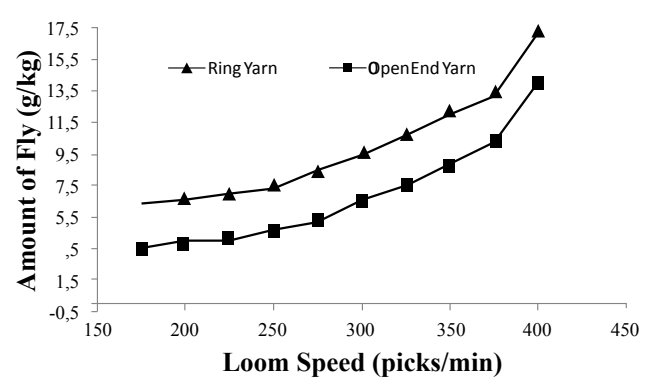

Figure 5: Effects of Spinning Types on Fiber Fly Generations. 


\begin{tabular}{|c|c|c|c|c|c|c|}
\hline \multicolumn{2}{|c|}{ Sources of Variances } & \multirow{2}{*}{\begin{tabular}{|l|} 
Sums of \\
Squares
\end{tabular}} & \multirow{2}{*}{\begin{tabular}{|l} 
Degree of \\
Freedom
\end{tabular}} & \multirow{2}{*}{\begin{tabular}{|l|}
$\begin{array}{l}\text { Mean } \\
\text { Square }\end{array}$ \\
1.48 \\
\end{tabular}} & \multirow{2}{*}{\begin{tabular}{|l} 
F Value \\
2.09 \\
\end{tabular}} & \multirow{2}{*}{$\begin{array}{l}\text { F Critical } \\
\mathrm{F}_{0.05,2,287}=3.84\end{array}$} \\
\hline \multirow{3}{*}{$\begin{array}{l}\text { Main } \\
\text { Factors }\end{array}$} & Yarn Count $(Y N)$ & & & & & \\
\hline & Yarn Type (YT) & 931.68 & 1 & 931.68 & 1312.22 & $F_{0.05,1,287}=2.60$ \\
\hline & Twist Factor (TF) & 1712.85 & 3 & 570.95 & 804.15 & $F_{0.05,3,287}=2.60$ \\
\hline \multirow[t]{4}{*}{ Interactions } & $\begin{array}{l}\text { Yarn Count-Yarn } \\
\text { Type }\end{array}$ & 67.88 & 2 & 33,94 & 47.80 & $F_{0.05,2,287}=2.60$ \\
\hline & $\begin{array}{l}\text { Yarn Count-Twist } \\
\text { Factor }\end{array}$ & 62.32 & 6 & 10.39 & 14.63 & $F_{0.05,6,287}=2.60$ \\
\hline & $\begin{array}{l}\text { Yarn Type-Twist } \\
\text { Factor }\end{array}$ & 58.89 & 3 & 19.63 & 27.65 & $F_{0.05,3,287}=1.88$ \\
\hline & $\begin{array}{l}\text { Yarn Count-Yarn } \\
\text { Type- Twist Factor }\end{array}$ & 44.43 & 6 & 7.41 & 10.44 & $F_{0.05,6,287}=1.88$ \\
\hline \multicolumn{2}{|l|}{ Error } & 186.04 & 264 & 0.71 & & \\
\hline \multicolumn{2}{|l|}{ Total } & 3067.05 & 287 & & & \\
\hline
\end{tabular}

Table 2: Analysis of variance (ANOVA) for fiber fly generation.

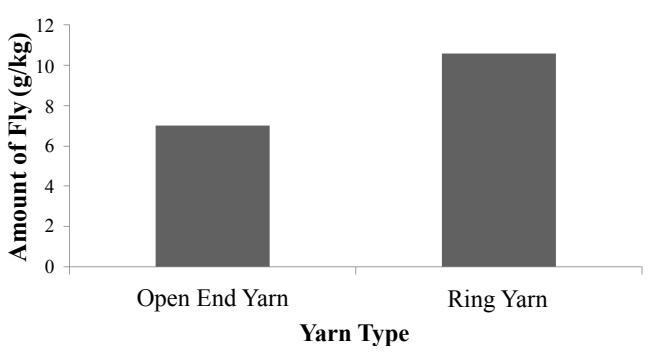

Figure 6: Effects of Twist Level on Fiber Fly Generations.

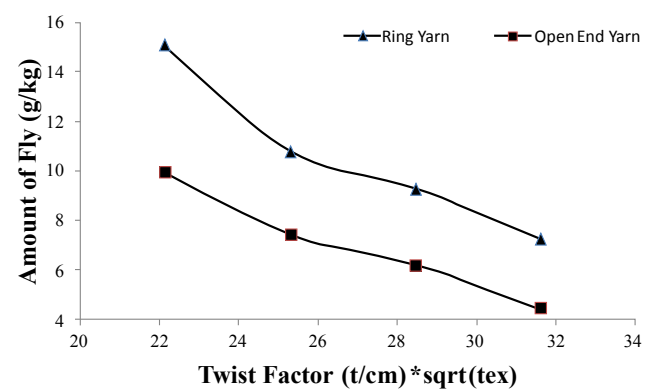

Figure 7: Effects of Yarn Linear Density on Fiber Fly Generations.

amount of the fiber fly decreases for the ring yarn as the linear density of the yarn increases due to the fact that the coarser yarns contain more fibers throughout their cross section as expected and reported by the previous studies $[5,2,3,12]$. However, the amount of the fiber fly increases as the linear density of the yarn increases for open end yarns as seen in figure 7 . As the linear density of the open end yarn increases, the wrapper fiber's wrapping height decreases for a given wrapper fiber's length. This will reduce the numbers of coils wrapping around the yarn which results more hairiness on the yarn surface. Therefore, the amount of the fiber fly increases for the coarse open end yarns. The statistical analysis, however, indicated that the effect of yarn count was not as strong as the other two parameters, namely, the spinning type and the twist factor on the amount of the fiber fly generation.

\section{Fiber fly distribution along the weft yarn path}

During the weaving operations, the speed of weft yarns is up to $1000 \mathrm{~m} / \mathrm{min}$. The weft yarns follow a particular path at which there are many contact points between the yarn and the machine parts. The fiber fly generation occurs while the yarn travels along its path and particularly at the first point where the weft yarn began to be unwound at which the yarn slides over the package. After this first stage, the fiber fly generation continues at the other points along its ways. Figure 8 shows the amount of the fiber fly collected from the different parts of the weaving machine. As seen on the figure, the maximum fiber fly generation was collected at the weft selection part just before the weft insertion point because this point is the hardest contact point at which weft yarn slides over the metal parts. Furthermore, during this hard movement, the loose fibers either are broken or are shed, which generates the fiber fly. The second point at which the most fiber flies generation area is the weft accumulator unit just before the weft selection point. The rest of the fiber fly was distributed between the unwinding section and the weft insertion point. This suggests that most of the fibers protrude from the yarn surface have been shed at the unwinding point. It is reasonable to have a large amount of the fiber fly at the unwinding section due to the fact that there is a positive tension on the yarn in addition to the frictional force among the yarns. The type of the yarn package influences the amount of the fiber fly generated at the unwinding section. The package conicity is a factor affecting how easily the yarn can be unwound from the yarn cone. The dimension and conicity of cones used in the experiment were commercially available cones in the market. As the conicity increases, the amount of the fiber fly at the unwinding section decreases as indicated in figure 9. As seen in the figure, there is a sharp drop on the fiber fly generation after $3^{\circ} 30^{\prime}$ because of the fact that the frictional force between the sliding yarn and the cone decreases (Figure 10).

\section{Conclusion}

The fiber fly generation is getting more attention because of the legal regulations requiring a clean working environment for

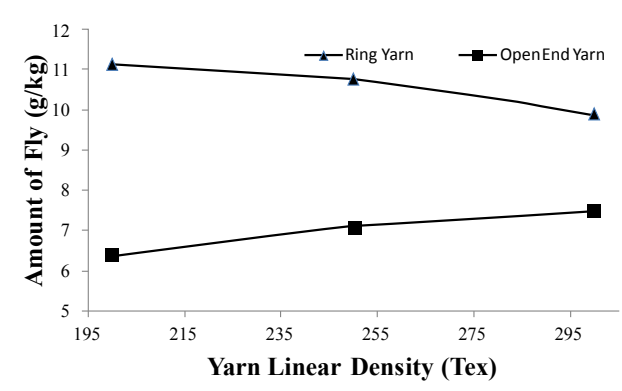

Figure 8: Relative Fiber Fly Distribution in Each Sector of Loom.

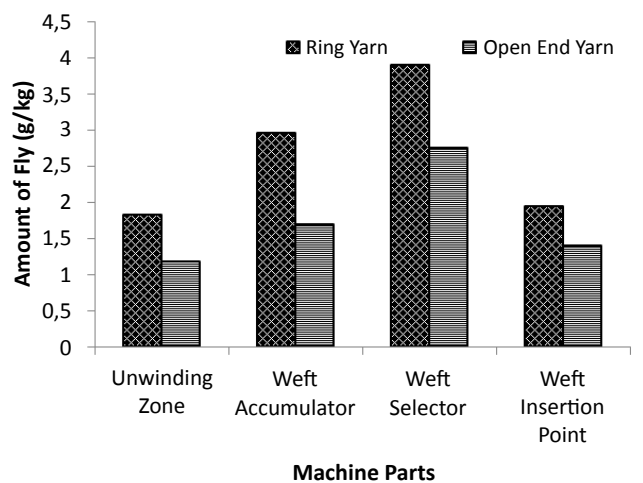

Figure 9: Effects of Package Conicity on Fiber Fly Generations. 


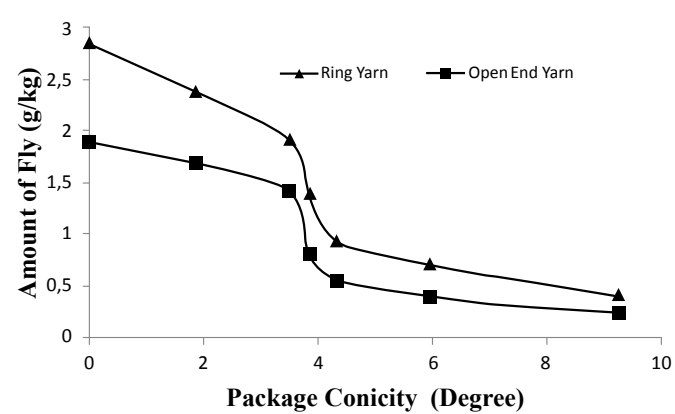

Figure 10: Drop on the fiber fly generation after $3^{\circ} 30^{\prime}$.

employees. The elimination of the fiber fly at the working environment is also necessary for a high efficiency and lower defective products for the companies in order to be able to get more competitive strain for today's competitive markets. The fiber fly is related to the physical properties of the fiber and machine parameters. As emphasized in the paper, longer mean length in the fiber length distribution reduces the amount of the fiber fly generated during the weaving operations. It was found that as the fiber linear density increases, the amount of the fiber fly slightly increases. The spinning type was another important factor affecting the fiber fly generation. The open end yarn is giving the less amount of the fiber fly than the ring yarns for the soybean fiber used. It was also noticed that as the twist factor, moisture content, and package conicity of the yarn increase, the amount of the fiber fly decreases for ring and open end yarns. Furthermore, as the loom speed increases, the amounts of the fiber fly also increases due to the fact that at higher loom speed, the fast yarn movement damages the yarn surface more severely. The fiber fly increases as the linear density of the open end yarn increases; however, this relationship is reverse for the ring yarns. Finally, the fiber fly distribution according to the weaving machine part revealed that the maximum amount of the fiber fly was detected on the weft selector section of the loom while the least amount of the fiber fly was detected on the unwinding zone. In order to reduce the fiber fly problem at the working environment, some precautions, such as suction units are to be installed to the different parts of the machines besides the acclimatization of the entire house. Even though, this will not reduce the total amount of the fiber fly generated from the yarn, but this will help to keep the working environment cleaner and safer for the employees.

\section{References}

1. Xu W, Xia Z, Wang X, Chen J, Cui W, et al. (2011) Embeddable and locatable spinning. Text Res J 81: 223-229.

2. Yuksekkaya E (2008) A study of fly generation during raising. J Text I 99: 169176.

3. Yuksekkaya ME (2010) Fiber fly Generation of $100 \%$ cotton yarns during warp preparation. J Text I 101: 270-275.

4. Brown P (1978) A Preliminary Study of the Fiber-Length Distribution in Fly Produced During the Weft Knitting of Cotton Yarns. Text Res J 48: 162-166.

5. Lawrence CA, Mohamed SA (1996) Yam and Knitting Parameters Affecting Fly During Weft Knitting of Staple Yarns. Text Res J 66: 694-704.

6. Lee JR, Ruppenicker GF (1978) Effects of Processing Variables on the Properties of Cotton Knitting Yarns. Text Res J 48: 27-31.

7. Ruppenicker GF, Lofton JT (1979) Factors Affecting the Lint Shedding of Cotton Knitting Yams. Text Res J 49: 681-685.

8. Pillay KPR (1964) A Study of the Hairiness of Cotton Yarns Part II: Effect of Processing Factors. Text Res J 34: 783-791.
9. Buhler G, Rieder O, Haussler W (1987) The Origins of Fiber Fly on Knitting Machines and Ideas for Reducing their Harmful Effect upon Knitting-Efficiency. Knitting Tech 9: 250-258.

10. Buhler G, Rieder O, Haussler W (1988) Fiber Fly; A Serious Problem for the Knitting Industry. Knitting Tech 10: 163-166.

11. Buhler G, Rieder O, Haussler W (1990) The Reduction of Fiber Fly by Obtaining the Best Possible Results from the Knitting Yarns. Knitting Tech 12: 35-39.

12. Yuksekkaya ME (2010) Fiber Fly Generation of $100 \%$ Acrylic Yarns during Weaving. Text Res J 80: 508-515.

13. Barella A, Torn J, Vigo JP (1971) Application of a New Hairiness Meter to the Study of Sources of Yam Hairiness. Text Res J 41: 126-133.

14. Goswami BC, Anandjiwala RD, Hall DM (2004) Textile Sizing. Marcel Dekker Inc. New York, USA.

15. Barella A (1966) The Hairiness of Yarns: A Review of the Literature and a Survey of the Present Position. Journal of the Textile Institute Transactions 57: T461-T489.

16. Subramanian TA, Grover JM, Salhotra KR (1971) A contribution to the Study of the Hairiness of Ring-Spun Yarns. J Text I 62: 424-437.

17. Knapton JJF (1972) Knitting of High Quality Double Knit Cloth: Part 2. Knitting Times 41: 44-49.

18. Montgomery DC (2005) Design and Analysis of Experiments. (6th edn) John Wiley and Sons, New York, USA. 